\title{
PREFACE: HEAT TRANSFER AND FLUID FLOW IN MICROSCALE PHENOMENA
}

With the rapid development of micro- and nanosystems, the interfacial phenomena at the fluid/solid interfaces, and also at the interfaces between two fluid phases, become more and more important. The decrease in systems' sizes results in an increase of surface over the volume effects compared with conventional systems. Shrinking of the systems' scales can be used successfully for heat transfer enhancement. However, modeling of these phenomena requires detailed knowledge of the velocity, as well as the temperature boundary conditions. The velocity slip and the temperature jump observed at the wall in the slip flow regime strongly depend on the nature and state of the wall, which are related to the nature of the fluid. Thus, the roughness of the wall and the chemical affinity between the wall and the fluid, although often not properly known, could strongly affect the fluid flow and heat transfer. In addition, when the characteristic scales of devices shrink, the ratio between the molecular mean-free path and the characteristic dimension of the problem can become large, and thermodynamic disequilibria appear on the boundary because the gas molecules do not have enough collisions to reach an equilibrium state.

There is a huge demand for effective thermal management of high-power devices, and two phase flows are actively studied for these applications. Recently, there also has been significant demand for the better understanding of phase change processes in combination with micro- and nanoengineered surfaces. The interfacial issues on the microscale are of key importance in relation to the newest heat transfer systems. The present special issue includes eight selected papers covering experimental, theoretical, and numerical approaches to the interfacial phenomena in micro- and nanosystems. These papers should help us work together toward progress in the field of heat transfer and in developing applications for energy and sustainability.

Last but not least, we would also like to express our gratitude to Professor Oleg Kabov, Editor-in-Chief, for his advice and to the Begell House staff for providing invaluable editorial assistance.

\section{Guest Editors:}

Irina Graur Martin

IUSTI Laboratory UMR CNRS 7343, Aix Marseille University, Marseille, France

\section{Huihe Qiu}

Department of Mechanical and Aerospace Engineering, The Hong Kong University of Science \& Technology, Clear Water Bay, Kowloon, Hong Kong

\section{Deborah Pence}

Faculty of Mechanical Engineering, School of Mechanical, Industrial, and Manufacturing Engineering, Oregon State University, Corvalis, OR

\section{Koji Takahashi}

Department of Aeronautics \& Astronautics, Kyushu University, Motooka, Nishi-ku, Fukuoka, Japan 